\title{
Feminismos Periféricos
}

$\begin{array}{ll}\text { Nombre: } & \text { Pilar Rodríguez Martínez (*) } \\ \text { Universidad: } & \text { Universidad de Almería } \\ \text { Ciudad: } & \text { Almería } \\ \text { País: } & \text { España } \\ \text { Correo: } & \text { pilarr@ual.es }\end{array}$

\section{Resumen}

En este artículo presentamos una propuesta conceptual, la de "Feminismos periféricos", que pretende englobar algunos de los planteamientos realizados en el marco de lo que se denomina feminismos de la tercera ola, feminismos post-coloniales o feminismos del tercer mundo. Se trata de un instrumento de análisis concebido para comprender mejor cómo se relacionan -o se pueden relacionar- las categorías de sexo, clase y raza (y etnicidad) en diferentes campos de análisis, momentos históricos y distintas disciplinas. Las protagonistas que dan sentido a esta propuesta son las mujeres que pueblan la periferia social: mujeres pobres, discapacitadas, con otra orientación sexual y, especialmente, las denominadas inmigrantes.

\section{Palabras claves}

Feminismos periféricos, tercera ola de feminismos, feminismos del tercer mundo, teoría postcolonial.

\section{Peripheral Feminisms}

In this paper we present the concept of "peripheral feminisms," which seeks to encompass the views of some women in the context of what is called the Third Wave of Feminism, Post-colonial feminisms, or Third World Feminisms. This concept is an analytical tool designed to better understand how we relate -or could relate-the categories of gender, class and race (and ethnicity) in different contexts, historical moments, and across different disciplines. Protagonists of Peripheral Feminisms are the women who populate the social periphery: poor women, disabled women, those with a different sexual orientation, and especially immigrant women.

\section{Keywords}

Peripheral Feminisms, Third Wave Feminism, Third World Feminisms, Post-colonial Theory.

(*)Doctora en Sociología. Profesora Titular de Sociología en la Universidad de Almería (España). Sus líneas de investigación son género y migraciones. 


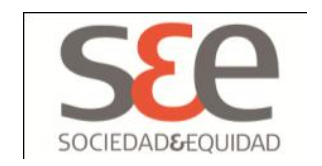

\section{Introducción}

En el presente artículo presentamos un análisis particular sobre las actuales agrupaciones de mujeres y feminismos. ${ }^{1}$ Ofrecemos, en primer lugar, un diagnóstico sobre lo que a nuestro juicio ha sucedido en el panorama occidental desde los años sesenta en adelante, resaltando algunos éxitos y fracasos de la segunda ola de feminismos. En segundo lugar, daremos paso a la descripción de las nuevas protagonistas y los nuevos proyectos feministas, que tienen mucho que ver con las problemáticas concretas que, en la actualidad, experimentan las mujeres en los márgenes sociales. Por último, presentaremos algunos de los rasgos que, a nuestro juicio, caracterizan a estos feminismos periféricos. Comenzaremos, pues, con nuestro diagnóstico.

Los feminismos de la segunda ola, tanto los de la igualdad (liberal, socialista) como los de la diferencia (radical, psicoanalítico o postestructuralista), contribuyeron a cuestionar la división social entre hombres y mujeres, tanto en su vertiente teórica como práctica. Sin embargo, asumieron un concepto de patriarcado que inducía a borrar las diferencias entre mujeres, en orden a salvaguardar para el análisis -y para la lucha- dos categorías sociales exclusivas y claramente delimitadas: hombres y mujeres.

Las narrativas de la igualdad y la diferencia insistían en la necesidad de terminar con los privilegios de los hombres sobre las mujeres, de manera que todas las mujeres -por el mismo hecho de serlo - debían compartir los mismos intereses. Las discusiones de los años setenta y ochenta en el seno de los movimientos feministas rondaron en torno a cuál era la institución social que había que

\footnotetext{
${ }^{1}$ El concepto de 'feminismos periféricos' apareció por primera vez en el marco de mi tesis doctoral (Rodríguez, 2002). Surgió como una herramienta que me permitía clasificar textos feministas que no partían de la idea de que mujeres y hombres constituyen dos grupos sociales homogéneos. Aunque en un inicio la propuesta conceptual fue formulada para realizar un análisis sociológico de las identificaciones sociales de sexo-género de las mujeres migrantes, con el paso del tiempo he ido integrando a otras actoras sociales y ampliando la bibliografía. Este texto se empezó a gestar tras una conversación con Silvia Pedraza, que me sugirió ampliar y poner en forma de artículo las ideas que aparecían en la introducción del libro que yo misma había editado sobre Feminismos Periféricos. El texto final también se ha beneficiado de las discusiones que han surgido en algunas conferencias que he impartido durante los últimos años en diferentes lugares de España, entre las que me gustaría resaltar la impartida en el Ciclo de Conferencias sobre Igualdad, organizado por el CEP en Granada, el 9 de marzo de 2009; en las Jornadas de la Facultad de Pedagogía de la Universidad de Barcelona, Dona, inmigració i prostitució: Claus per comprendre la realitat, el 30 de abril de 2009; en la Escuela de Verano sobre Feminismos Postcoloniales, que se celebró en Granada el 3 de julio de 2009 organizada por el Master Erasmus Mundus Gemma; en las VII Jornadas Formativas de Mujer y Discapacidad, que se celebraron el 22 de octubre de 2009 en Toledo, organizadas por la Confederación Española de Personas con Discapacidad Física y Orgánica (COCEMFE); y la que dicté en el marco del seminario del Instituto de Investigaciones Feministas Feminismos y cambio social, celebrado el 7 de octubre de 2010 en Madrid. Me gustaría agradecer a las organizaciones y las personas que amablemente me invitaron a explicar la propuesta, especialmente a mis colegas Maite Martin Palomo y Margarita Birriel Salcedo, y a quienes se dejaron seducir por mis sugerencias. Estoy en deuda, sobre todo, con quienes plantearon críticas que me han permitido mejorar el planteamiento. Los/as revisores de la revista Equidad y Sociedad también han contribuido con sus observaciones a mejorar el texto.
} 


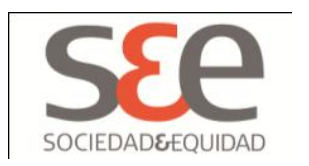

situar en el centro de la diana. Se suponía que apuntando hacia ella todas nuestras flechas al unísono podríamos ganarle la batalla al patriarcado: las radicales pusieron el ojo en la familia; las marxistas-socialistas en el mercado de trabajo; las liberales en la esfera política; las psicoanalistas en la mente; y las postestructuralistas en la cultura. Aquellos debates sobre igualdad frente a diferencia, o sobre la relevancia de una de esas instituciones en comparación con las otras, hicieron que se abarrotaran las salas de conferencias y que se derramaran ríos de tinta en una época en la que, también, se asistía a un auge de la presencia del movimiento feminista en la calle y en los medios de comunicación.

Lo que ocurrió en España -unos años después que en otros países occidentales - fue que, tanto en la calle como en los medios de comunicación, las campañas que consiguieron una mayor unanimidad fueron las relacionadas con las reformas legales - desde la ley del divorcio, la del aborto, pasando por la reforma del código civil hasta llegar a la ley sobre la violencia de género - y con la intervención del Estado para evitar las discriminaciones que muchas mujeres estaban sufriendo. Sin embargo, el desarrollo de aquella habilidad colectiva para exigir cambios en las leyes hizo que algunas se quejaran de que, en la práctica, las reivindicaciones del movimiento feminista no habían llegado mucho más allá de las demandas del feminismo liberal que ya planteara Betty Friedan en La mística de la feminidad. ${ }^{2}$

A los cambios legislativos - por cierto, nunca saldados por los gobiernos al gusto de las feministas-, hay que sumar la aparición de lobbies de mujeres en la lucha por ganar espacios en las instituciones públicas y en los partidos políticos. De manera que, las luchas por el poder político han llevado a unas pocas mujeres a ocupar espacios de poder anteriormente reservados a los hombres, pero eso no se ha traducido en una mayor presencia de mujeres de las clases medias organizadas en los movimientos feministas, sino más bien todo lo contrario. $\mathrm{Ni}$, por supuesto, se ha avanzado en la incorporación al movimiento organizado de las mujeres de las clases sociales bajas. Asistimos en nuestros días al entronamiento de unas cuantas académicas y políticas, algunas de las cuales miran hacia abajo -a un amplio sector de mujeres de niveles educativos medios y altos e insertas en el mundo laboral, que formaron parte del movimiento en otros tiempos - únicamente para legitimar unas actuaciones que, desde hace tiempo, ni siquiera conectan con esas supuestas 'bases'. Este es el escenario en el que, a finales del siglo XX, se ha empezado a percibir públicamente la presencia de los feminismos de la tercera ola, feminismos post-coloniales y/o feminismos periféricos.

\footnotetext{
${ }^{2}$ Como señalan Patricia Madoo Lengermann y Jill Niebrugge-Brantley, en el caso norteamericano este feminismo se ha venido compaginando muy bien con las políticas públicas, "el feminismo liberal (...) está en la base de una buena parte de la literatura popular que aboga por la mujer profesional, por la igualdad de responsabilidad ante los hijos y por la necesidad de una enseñanza igual para niños y niñas; guía muchas de las políticas iniciadas por el movimiento (...). El feminismo liberal y la corriente principal de las creencias políticas estadounidenses se complementan fácilmente, y ello ayuda a comprender la popularidad de esta variante de teoría feminista" (Madoo Lendermann y Niebrige-Brantley, 1993: 273).
} 


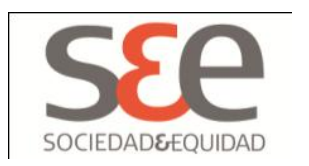

\section{Otras protagonistas y otros feminismos}

La heterogeneidad de las mujeres empezó a manifestarse en el escenario público en el mundo occidental a finales de los ochenta. ${ }^{3}$ Eran de destacar junto a las lesbianas, ${ }^{4}$ las mujeres mayores, las prostitutas, transexuales, discapacitadas, jóvenes, negras, gitanas, de otros grupos étnicos, migrantes y mujeres del Tercer Mundo. Estas nuevas actoras sociales tomaron la iniciativa, cuestionando algunos de los lugares comunes de las feministas occidentales, jóvenes, sin discapacidades, blancas, heterosexuales y de clase media. Quizá se hayan producido cambios cualitativos en los sentimientos de malestar o quizá asistimos a la creación de nuevas agrupaciones, que intentan responder al reto de vivir en un mundo más o menos des-centrado, fracturado y sin sentido. En todo caso, esta proliferación de identificaciones ya ha conseguido hacerse eco en los movimientos feministas y en las perspectivas de análisis. Los denominados 'estudios de mujeres', que tradicionalmente se ocupaban de la división social entre los sexosgéneros, han pasado a considerar como problema central las 'diferencias entre las mujeres', hasta tal punto que autoras como Sandra Harding consideraron que la cuestión del 'feminismo en la ciencia' se había retomado, planteando la cuestión de 'la ciencia en el feminismo' (Harding, 1993).

Afirmar la heterogeneidad de las mujeres implica reclamar "la ciencia en el feminismo", lo que lleva -a la larga- a cuestionar la idea de que la sociedad está divida únicamente en dos grupos sociales coherentes. De modo que, a partir de los noventa, los nuevos planteamientos ${ }^{5}$ no perseguirán encontrar el eje común de opresión, dominación, subordinación o explotación del hombre sobre la mujer. No buscarán tampoco descubrir el motor del patriarcado, que podría explicar la situación de todas las mujeres. No defienden que pueda existir a priori una identidad

\footnotetext{
${ }^{3}$ En el caso español, la 'diversidad' de grupos de mujeres se pudo constatar en las Jornadas organizadas por la Coordinadora de Organizaciones Feministas del Estado español en 1993. Los diferentes talleres que se organizaron a propuesta de los grupos incluían, al lado de los esperados como 'reparto de tiempos' y 'nuevas políticas de empleo', asuntos tan 'exóticos' como 'merensalsa' o 'teología de la liberación', al lado de las conferencias sobre teorías feministas por parte de las académicas. Lo curioso es que los temas 'exóticos' reunieron e ilusionaron a la mayor cantidad de público allí presente.

${ }^{4}$ Las lesbianas, como grupo 'minoritario' en el colectivo de mujeres feministas organizadas, no constituyen un grupo nuevo. Desde los setenta, en las organizaciones se discutía y se trataron de dar respuestas a los planteamientos de estas mujeres. 'El lesbianismo como opción política' planteó que todas las mujeres feministas -fueran heterosexuales o lesbianas- debían reivindicarse 'públicamente' como lesbianas. Aún así, muchas mujeres siguieron planteando que tanto en los objetivos de las campañas como en las teorizaciones predominaban los problemas y planteamientos teóricos que sólo hacían referencia a la familia nuclear heterosexual.
}

${ }^{5}$ Frente a esos nuevos planteamientos, las feministas -tanto las de la igualdad como las de la diferenciareclamarán la universalidad de los géneros, y, por tanto, la posibilidad de establecer unos objetivos comunes entre, por ejemplo, mujeres del Primer y Tercer Mundo, blancas y negras, de diferentes estratos sociales, de diferentes opciones sexuales y de diferentes edades. Se reclama que, aunque tras las distintas perspectivas se pueda encontrar un grupo de mujeres particular, las diferentes posiciones deberían tener en cuenta que sus intereses feministas podían ser compartidos por las otras. Que, en todo caso, es una cuestión de énfasis. 


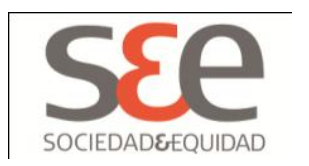

unificada ni unos intereses compartidos. Descubriendo y reconociendo la diversidad, heterogeneidad e incluso el conflicto entre las mujeres, se apoyarán en problemáticas que cuestionan la identidad genérica sostenida por feministas blancas, mayores, sin discapacidades, del Primer Mundo, heterosexuales y de clase media. Y, en vez de defender políticas de identidad, propondrán compromisos concretos con los que identificarse, que no siempre suponen avances en la balanza de poder entre hombres y mujeres.

Estos planteamientos críticos se alzan en dos frentes: por una parte, nos vamos a encontrar con los feminismos postmodernos, que niegan la posibilidad teórica de una identidad de género unificada y esencializadora (Butler, 1990, 2000 y 2001); y, por otra parte, hallaremos propuestas que plantean las desigualdades entre mujeres como punto de partida, y defienden que las alianzas en y entre los grupos deben hacerse manteniendo también criterios como la edad, la discapacidad, la clase social, la raza, la nacionalidad o la opción sexual. Si atendemos a la raza y/o nacionalidad, las propuestas más sugerentes parecen provenir de los feminismos post-coloniales que pretenden, por un lado, racializar la teoría feminista dominante $y$, por otro, introducir los asuntos feministas en las conceptualizaciones sobre el colonialismo y post-colonialismo (Lewis y Mills, 2003: 3). Racializar la teoría dominante significa ocuparse del análisis de las relaciones entre las mujeres occidentales $y$, hablando en términos generales, de las mujeres indígenas. El concepto de feminismos periféricos pretende ser una herramienta que sirva para entender las experiencias de esas mujeres indígenas.

Así que denominamos feminismos periféricos a los planteamientos que, desde los años ochenta y, sobre todo, en los noventa, ponen en cuestión los planteamientos de las feministas del centro (occidentales, blancas, heterosexuales, jóvenes, sin discapacidad y de clase media). Entre estos planteamientos, tenemos a las que reclaman específicamente una presencia en la esfera pública de las 'mujeres del Tercer Mundo' como categoría social (Truth, 1851; Sandoval, 1991; Göle, 1991; Mernissi, 1991; Talpade Mohanty, 1991; Anzaldúa, 1993; Mirza, 1997; Spivak, 1999; Lugones, 1999; Moghissi, 1999; Haraway, 1999; Afshar y Maynard, 2000; Oyewumi, 2000; Flores, 2000; Bannerji, 2000; Afary, 2000). En Europa y Estados Unidos esa categoría social engloba a las mujeres que, en sentido amplio, habitan o proceden del Tercer Mundo: mujeres afroamericanas, negras, 'mujeres de color' o 'inmigrantes'. En el caso de Estados Unidos, se suele usar el término women of color en sentido general para referirse a estas mujeres. En el caso del Reino Unido se suele hablar de black women o de asian women. En general, se habla de third world women (Bannerji, 2000: 537-560). En la Europa del Sur actual tendríamos que mencionar, sobre todo, a las mujeres 'inmigrantes'.

Con este término pretendemos aludir a las mujeres que provienen de países pobres, africanas, latinoamericanas, asiáticas y de Europa del Este. Además, retomamos el concepto de 'mujeres del tercer mundo' cuando alude también a una 'comunidad imaginada', es decir, a un modo de pensar sobre la raza, la clase social y el género, que destaca su carácter relacional (Talpade Mohanty, 1991). Pero además hay que incluir a quienes consideran que por razón de edad, de opción sexual, por ser discapacitadas o por pertenecer a las clases bajas u otros grupos étnicos, no 


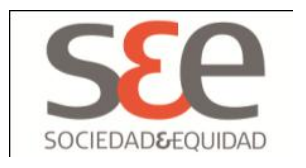

se encuentran representadas en el concepto de mujeres que mantienen las feministas del centro (Morris, 1993, 2008; Shakespeare, 1996; Peters, 1996). Como señala Jenny Morris, una de las feministas discapacitadas:

Las experiencias de las mujeres blancas de la clase media han sido cogidas como norma y las experiencias de las otras mujeres han sido tratadas como 'diferentes', como un sujeto de estudio y análisis particular. Así, la realidad de las mujeres blancas de clase media es la base de la teoría y del análisis general (lo mismo que lo fue la realidad de los hombres) y la realidad de los otros grupos de mujeres es tratada como particular, como separada de lo general.

(Morris, 1993: 58)

De manera que los feminismos periféricos no se definen sólo por la base biológica, cultural o nacional de las personas que los defienden, sino que también es posible posicionándose con ellos en -o respecto a- un contexto social. Explícitamente, se está aludiendo a un 'contexto común de lucha'. ${ }^{6}$ En síntesis, el término 'feminismos periféricos' alude directamente a localizaciones geográficas de origen, pero también, y sobre todo, a posicionamientos en torno a coyunturas sociohistóricas concretas, reales y construidas.

\section{Reconstruyendo la historia desde los feminismos periféricos}

Los feminismos periféricos toman como punto de partida histórico el tono del discurso de Sojourner Truth, ${ }^{7}$ que en su famoso discurso 'Ain't I a woman?', pronunciado en 1851, durante una convención sobre derechos de las mujeres compuesta casi exclusivamente por mujeres blancas, se expresaba de este modo:

En fin, hijas mías, aquí se está haciendo tanto ruido que está claro que hay algo que no funciona. Creo que muy pronto el hombre blanco va a tener problemas, pues tanto las mujeres del norte como los negros del sur ya están hablando de derechos. Pero, ¿̇de qué va todo esto? Aquel hombre de allí dice que a las mujeres se les debe ayudar a subir a los carruajes, que deben ser cogidas en brazos cuando cruzan las zanjas y que se les debe ceder el mejor sitio en todos lados. ¡A mí nadie me ayuda nunca a subir a los carruajes, o a

\footnotetext{
${ }^{6} \mathrm{Al}$ decir que no es condición necesaria ser negra, o 'inmigrante' para etiquetarse como 'mujer del tercer mundo’ se está asumiendo la defensa postmoderna de desregulación del sujeto que es una de las maneras más efectivas de huir de esencialismos.

${ }^{7}$ Sojourner Truth, había sido esclava desde su nacimiento en el estado de New York sobre 1797. Fue liberada en 1827 cuando en New York llegó la legislación antiesclavista. Llegó a ser una abolicionista activa y una de las oradoras más famosas de su tiempo.
} 


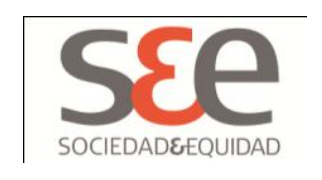

saltar los charcos, ni me cede el mejor sitio en ningún lado! ¿Y es que yo no soy una mujer? ¡Miradme! iMirad mi brazo! ¡He arado, sembrado y cosechado, y ningún hombre lo habría hecho mejor! iYo podría trabajar y comer tanto como un hombre -si tuviera qué comer-y soportar los latigazos también! ¿Y es que yo no soy una mujer? He parido trece niños y he visto cómo vendían a la mayoría de ellos como esclavos. ¡Me he desgarrado chillando como una madre y nadie, excepto Jesús, pudo oírme! ¿Y es que yo no soy una mujer? Y, luego... hablan de eso que se tiene en la cabeza, ¿cómo dicen que se llama? (Un miembro de la audiencia susurra: 'intelecto'). Eso es, encanto, ¿qué tiene eso que ver con los derechos de las mujeres o de los negros? (...) Aquel hombre bajito de traje negro dice que las mujeres no pueden tener tantos derechos como los hombres, iporque Cristo no era mujer! ¿De dónde vino tu Cristo? ¡De Dios y de una mujer! iLos hombres no tuvieron nada que ver con él! Si la primera mujer que Dios hizo tuvo fuerza suficiente para darle la vuelta a la tortilla, todas estas mujeres juntas deberían ser capaces de darle la vuelta de nuevo y poner las cosas en su sitio definitivamente. Ahora piden hacerlo. Deberían dejar que lo hagan. Muchas gracias por haberme escuchado. La vieja Sojourner no tiene ya nada más que decir.

(Truth, 1851:1)

Los feminismos periféricos de la última década del siglo XX se caracterizan por poner énfasis en el carácter relacional del sexo-género, la raza (etnicidad) y la clase social. No reivindican a las sufragistas como antecesoras, sino -como en este caso - a una mujer negra que simboliza la lucha contra la esclavitud en Estados Unidos. El discurso de Truth muestra, ejemplarmente, lo que se está reclamando: el contraste entre un análisis de sexo-género centrado en la división entre hombres y mujeres (blancas), con otro discurso que se articula en torno a las relaciones entre mujeres (negras) y hombres - y mujeres - (blancos). Y es que la discriminación de género no es adicional, sino relacional, lo que significa que colorea y es coloreada por otras divisiones sociales, como la clase social y la raza (o etnicidad).

Lo interesante del discurso de Truth es que nos está hablando de un tipo de feminidad cualitativamente diferente al de las mujeres blancas de su tiempo. Parece haber acudido a apoyar una convención de mujeres -blancas-, pero tiene serias dudas respecto a la universalidad de los intereses de las mujeres en tanto que colectivo, por lo que pregunta a la audiencia si es que ella no es una mujer. Es un discurso semejante al tipo de disertación que Audre Lorde llevara a cabo en 1983 en un congreso en el que duda de la potencia revolucionaria de los feminismos hegemónicos. Algo después de un siglo, la decepción respecto a la unanimidad de intereses en el colectivo de las mujeres parece ser incluso mayor: 


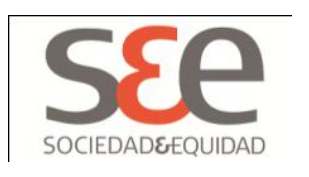

Quienes nos mantenemos firmes fuera del círculo de lo que esta sociedad define como mujeres aceptables; quienes nos hemos forjado en el crisol de las diferencias, o, lo que es lo mismo, quienes somos pobres, quienes somos lesbianas, quienes somos Negras, quienes somos viejas, sabemos que la supervivencia no es un asignatura académica. La supervivencia es aprender a mantenerse firme en la soledad, contra la impopularidad y quizá los insultos, y aprender a hacer causa común con otras que también están fuera del sistema y, entre todas, definir y luchar por un mundo en el que todas podamos florecer. La supervivencia es aprender a asimilar nuestras diferencias y convertirlas en potencialidades. Porque las herramientas del amo nunca desmontan la casa del amo. Quizá nos permitan obtener una victoria pasajera siguiendo sus reglas del juego, pero nunca nos valdrán para efectuar un auténtico cambio. Y esto sólo resulta amenazador para aquellas mujeres que siguen considerando que la casa del amo es su única fuente de apoyo.

(Lorde, 2003: 118)

Para Lorde, como para Truth, el feminismo no tiene sentido sacándolo de su contexto racializado y de clase ( $y$, se podría añadir, fuera de las divisiones sociales basadas en la opción sexual, la edad, etc.); divisiones que, según nos dice, no podrán ser atendidas sin alterar cualitativamente el orden social existente. Para ella, el hecho de ser mujer no se puede separar del hecho de ser pobre, lesbiana, negra y mayor, instancias que a su juicio constituyen también lugares desde los que se pueden proyectar reivindicaciones feministas. Lorde avisa de que las que sólo tienen en cuenta la categoría sexo a la hora de articular sus reivindicaciones corren el peligro de estar usando las 'herramientas del amo'. Es decir, los feminismos que sólo aspiran a subvertir el orden social en lo que se refiere a hombres y mujeres no se preocupan por otras divisiones sociales que, para las mujeres de la periferia social no sólo son prioritarias, sino que además definen el grueso de su malestar, de su soledad, del sentimiento de impopularidad, por las que son insultadas y por la que sienten que están 'fuera del sistema'.

Y es que cuando se experimenta la desigualdad social basada en la clase social, la raza, la discapacidad, la opción sexual o la edad, se intuye que los cambios sociales necesarios para terminar con todas esas desigualdades sociales no se pueden llevar a cabo sin desordenar la posición social de las mujeres blancas, heterosexuales y de clase media, lo que sin duda dará lugar a conflictos entre mujeres; y también se descubre que, quizá, para avanzar en algunos de esos objetivos será necesario - también - contar con los hombres. A nuestro juicio, la constatación de estas luchas entre las feministas del centro y de la periferia no ha de leerse como un síntoma de decadencia de la capacidad de las mujeres para articular sus reivindicaciones -como les gustaría a 


\section{sfe \\ Sorem}

los anti-feministas-, sino todo lo contrario: como expresión de ciudadanía entre mujeres que discuten sobre quién y en qué condiciones está legitimada para representar al grupo.

Además, las herramientas del amo -el voto, las peticiones de cambios en las leyes en democracias paritarias, etc. - nunca llevarán a provocar, a juicio de Lorde, un cambio social de ese calado. En este aspecto, Lorde avisaba sobre un aspecto clave: la relación entre los cambios en las leyes y los cambios en la sociedad. $Y$ es que, como hemos podido comprobar en las democracias occidentales, el establecimiento de leyes igualitarias no ha traído como consecuencia un cambio real en las relaciones entre hombres y mujeres. $O$, dicho de otro modo, las leyes de igualdad entre los sexos-géneros (incluidas las leyes de discriminación positiva) que se han proclamado en los estados del bienestar, no han supuesto el fin de la violencia de género, ni han erradicado las desigualdades salariales, ni han impedido que sean las mujeres las que tienen que hacer frente a los cuidados -de los hijos-as, enfermos-as, etc.-. Han permitido que las mujeres puedan denunciar situaciones de violencia doméstica, pero no han terminado con esa violencia; han facilitado el que las mujeres puedan denunciar situaciones de discriminación salarial, pero no han erradicado la segmentación del mercado laboral según el sexo; han hecho que se proclamen políticas para conciliar la vida laboral y familiar, pero no se han redistribuido las tareas por lo que muchas sufren una sobrecarga de trabajos o han externalizado parte de las tareas domésticas y de cuidados mediante la contratación de mujeres migrantes como trabajadoras domésticas. Lo que parece que ha ocurrido es un cambio cualitativo en el tipo de orden social de sexo-género, que ha pasado de estar centrado en la coerción a centrarse en el consentimiento (Rodríguez, 2004). En este, como en otros aspectos, la igualdad ante la ley no comporta el fin de las desigualdades, sino una nueva articulación de las mismas.

\section{Importancia de la localización, interseccionalidad e identificaciones}

Los feminismos periféricos señalarán una y otra vez la importancia de la localización (Rich, 2003) y de no dar por supuesta ninguna identidad que no sea resultado de un análisis sociológico. ${ }^{8}$ A nuestro juicio, es en esta insistencia en la necesidad de situar el pensamiento, de enraizarlo en las prácticas cotidianas, donde se expresa la potencia de las aportaciones que se realizan desde la periferia. $Y$ es que producen análisis que intentan no dar por supuesta ninguna identidad previa -ninguna esencia- y que se centran en los efectos que producen determinadas maneras de aparecer -o de re-presentar a otras-como mujeres, y los cambios que operan en la identidad cuando se tiene en cuenta el contexto. ${ }^{9}$ Estos análisis reclaman que,

\footnotetext{
${ }^{8}$ Chandra Talpade Mohanty plantea este asunto con varios ejemplos de estudios que empiezan afirmando un tipo de identidad de las mujeres, antes de reunir los datos sobre ellas. Según la autora, de ese modo se pierde de vista la particularidad de cada grupo de mujeres, y no se puede desarrollar un análisis sobre las estructuras sociales concretas que dan lugar a ese tipo de identificación ni tampoco se pueden percibir las formas de resistencia que las mujeres desarrollan (Talpade Mohanty, 2003).

${ }^{9}$ Como señala Floya Anthias: "Soy más que una mujer en algunos contextos en comparación con otros, de igual modo que soy greco-chipriota o más que una socióloga en otros casos. Sin embargo, estas etiquetas no
} 


\section{SEe}

Cada persona se sitúa en el mundo en la intersecciones de varias categorías: es latina, mujer, baja, madre, lesbiana, hija, de ojos castaños, pelo largo, aguda, con mal humor, trabajadora, terca. En un momento determinado en el tiempo y en el espacio, alguna de esas categorías es central para su ser y para su habilidad de actuar en el mundo. Las otras no importan en ese momento. Algunas categorías como la raza, género, clase y la orientación sexual son importantes la mayoría de las veces. Pero otras veces apenas importan. Cuando algo o alguien subrayan una de sus categorías y la pone en el primer plano, puede considerarse como una persona dominante, opresora de las otras categorías. Otras veces, incluso la mayor parte de las veces, se oprime a sí misma. Puede tomar nota de lo que ha aprendido en un estatus subordinado y aplicarlo para bien o para mal cuando subraya una de sus categorías dominantes. Por ejemplo, habiendo sido maltratada como niña, puede llegar a ser una madre respetuosa o abusadora.

(Grillo, 1995:17)

En efecto, las relaciones entre el género, la clase y la raza pueden ser entendidas de varios modos. La primera, sería centrarse en una de las categorías, y derivar las otras de ella. Este sería el caso que nos están describiendo críticamente Truth y Lorde, en el que los feminismos blancos y de clase media tratan de reducir las luchas de las mujeres a la desigualdad de sexo-género. También, sería el caso de los marxistas que consideraban la clase social como la categoría central que daba cuenta del modo de producción capitalista. Según esta versión, la desigualdad entre hombres y mujeres constituye otra división social que, al igual que la raza, el capitalismo usa para obtener mayores beneficios. El problema de esta manera de relacionar las categorías sociales es que reduce las relaciones entre hombres y mujeres (y entre mayorías y minorías étnicas o raciales) a las relaciones que se producen en el modo capitalista de producción de bienes y servicios. $O$, en el primer ejemplo, reduce las desigualdades de clase, de raza, opción sexual, discapacidad y edad, a la desigualdad de sexo-género.

Una segunda manera de abordar la relación entre las categorías de sexo, clase y raza es considerar que es posible adicionar las desigualdades, de manera que afirmaríamos que las mujeres están discriminadas, doblemente discriminadas o triplemente discriminadas. Estarían discriminadas las mujeres de la burguesía (respecto a los hombres); las de la clase obrera estarían doblemente discriminadas (respecto a los hombres y a la burguesía); y las mujeres de la clase

son exactamente como diferentes sombreros coloreados que una puede ponerse o descartar. Son más bien como diferentes capas -o estratos- que pueden ponerse en un orden diferente -algunas arriba, algunas abajoen diferentes momentos" ( Anthias, 1998: 507). 


\section{s\&e \\ sconenowa}

obrera y de minorías étnicas o raciales estarían triplemente discriminadas (respecto a los hombres, a la burguesía y a la mayoría étnica o raza dominantes). De hecho, la idea de que las mujeres migrantes del sur están triplemente discriminadas sigue manteniéndose en muchos foros.

El problema de sumar discriminaciones es que las personas no experimentamos las divisiones sociales una tras otra. Nuestras experiencias no se viven de manera separada y luego se adicionan, sino que el conjunto nos ubica en situaciones sociales concretas donde los efectos de una división social pueden multiplicar o evitar los efectos de otra división social. Otro ejemplo, cuando en España se produce el estereotipo de las mujeres procedentes de los países del este de Europa como 'putas', las mujeres que proceden de esos países no experimentan una adición de opresiones. De golpe, y en muchas facetas de sus vidas, son discriminadas 'aparentemente' como mujeres. Sin embargo, las razones de esa discriminación que aparece como de género tienen que ver también con la clase social y con la nacionalidad de origen. Se es una 'puta', entre otras cosas, porque una mujer que proviene de un tercer país que no pertenece a la Unión Europea no puede ser enfermera, secretaria o ingeniera, sino 'puta'. 0 , en otros casos, se es 'puta' porque se mantienen relaciones sexuales con hombres españoles casados -o incluso solteros-, del exogrupo, por lo que se traspasa la frontera étnica. En algunos casos, se es una 'puta' simplemente porque se es joven y guapa. En la estructura social española actual, identificar a una mujer del este como 'puta' justifica el hecho de pagarle menos, no ofrecerle un buen puesto de trabajo, desaprobar una posible relación con un hombre 'de los nuestros' o no tenerla en cuenta como persona cabal y responsable.

Quienes defienden la adición de las opresiones no se dan cuenta de la dificultad que encuentran quienes sufren esas discriminaciones para encontrar un foro común con otras mujeres desde el que poder proyectar sus reivindicaciones. Suponen, sin más, que las mujeres de la periferia deberían poder conformarse con plantear sus reivindicaciones en un foro feminista y no parecen preocuparse por saber cuántas feministas blancas, de clase media, sin discapacidad, jóvenes y heterosexuales estarían dispuestas a abrazar luchas anti-globalización, contra las leyes de extranjería, antirracistas, obreras, que defiendan los intereses de las mujeres del Tercer Mundo, mayores, lesbianas, con discapacidades o transexuales: Cuantas, por ejemplo, estarían a favor de una política de apertura de fronteras que terminara de una vez por todas con los problemas de 'documentación' de la mujeres -y hombres- que provienen de países pobres?

Esas dificultades son las que se intenta afrontar en el terreno conceptual con nociones como la de posicionalidad translocalizacional. ${ }^{10}$ En el caso de las mujeres migrantes, como en el de las

\footnotetext{
${ }^{10}$ Así, "El género, el ethnos (etnicidad y 'raza') y la clase pueden ser vistos como intersecciones y espacios ontológicos que interactúan, que vinculan relaciones sociales y procesos sociales (que tienen dimensiones experienciales, intersubjetivas, organizacionales y representacionales) que se incorporan y se articulan en coyunturas particulares para producir resultados/efectos sociales estratificados. No se puede sostener ningún análisis en el nivel de los efectos/resultados sociales que se haga de manera aislada. La analogía de la rejilla puede ser útil cuando se aplica a los individuos. Las diferentes rejillas son experimentadas contextual y
} 


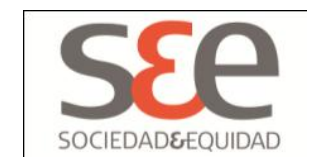

nativas, el estatus social se constituye a través de una complicada interacción entre clase, cultura, religión y otras instituciones y marcos de referencia (Mohanty, 2008:134). La interseccionalidad, se refiere específicamente a la interacción entre el género, raza y otras categorías de diferencia en los distintos individuos, prácticas sociales, disposiciones institucionales, e ideologías culturales y los resultados de estas interacciones en términos de poder (Cole, 2009).

En esa manera de hacer teoría, las feminidades se re-presentan de un modo más flexible e incierto. Aparecen como materias vivas que demandan un análisis sociológico que tiene que ser creativo si quiere llegar a captar su riqueza y ambigüedad. Pues se trata de adivinar algunos rasgos interesantes que traduzcan la sensualidad y el nomadismo que caracterizan a las actoras sociales de este tiempo. Así, frente al pensamiento plano de la modernidad, el de la abstracción, el cálculo, los números y las cantidades, las nuevas actrices postmodernas proyectan imágenes móviles y escurridizas de un mundo que, misteriosamente, sigue estando por inventar. En el terreno político, estos planteamientos y luchas son los que - ahora - sorprenden, deslumbran e ilusionan a cada vez más gente. No tendría que extrañar el hecho que se trate de análisis y luchas mucho más efímeras: aparecen y desaparecen, articulando una y otra vez diferentes desigualdades sociales que, en un momento dado, coinciden en luchas concretas. Y es que, en la dinámica social, las luchas de las mujeres no siempre suponen un avance en lo que se refiere a la relación de desigualdad entre hombres y mujeres. Cuando se pretende mejorar la posición respecto a una división social, es posible que se tenga que ceder -o renegociar, o apoyarse en otra. ${ }^{11}$ Los feminismos de la segunda ola habían asumido que existe una causalidad unilineal entre los atributos culturales, las identidades sociales y la conciencia social, unilinealidad difícil de sostener cuando se lleva a cabo un análisis histórico. ${ }^{12}$

situacionalmente como conjuntos de instancias discursivas simultáneas y mutuamente efectivas, y como prácticas sociales" (Anthias, 1998: 531).

${ }^{11}$ Ver, por ejemplo, la argumentación de Amalia Sa'ar en torno a lo que denomina 'liberal bargain' y que ejemplifica en el análisis sobre las mujeres de origen palestino, educadas, que viven en Israel (Sa'ar, 2005).

${ }^{12}$ A nuestro juicio, sería de mucho interés revisar las apuestas de las mujeres en cada época histórica para poder entender mejor cómo se relacionan las diferentes divisiones sociales. Por ejemplo, la apuesta de las mujeres españolas por la educación que ha tenido lugar desde los años setenta del siglo XX es posible que haya que entenderla no como un logro en lo que se refiere a la relación entre hombres y mujeres, sino como un avance en lo que tiene que ver con su posición de clase. Dicho avance ha supuesto, por ejemplo, el que las mujeres redujeran enormemente su fecundidad en aras a mejorar considerablemente su inserción en el mundo laboral y su disfrute de la sociedad del ocio. Como no se cuestionó la división de sexo-género, muchas de esas mujeres -buenas profesionales y sin hijos- siguen manteniendo los roles tradicionales en lo que se refiere a sus parejas (división de tareas, de responsabilidades en el hogar, etc). El nivel educativo no sería, por tanto, un buen indicador sobre la situación de igualdad/desigualdad entre los sexos-géneros. 


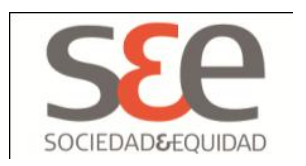

\section{Características de los feminismos periféricos}

Así pues, las identificaciones de sexo-género que defienden las 'colonizadas' son primordialmente relacionales e 'históricas'. $Y$, puesto que provienen de lugares donde no se sintieron con fuerza las consecuencias de la Revolución Francesa ni la Revolución Industrial, no siempre están centradas en lo que ocurre en la familia nuclear. En este sentido, hay que admitir que los avances que se consiguieron en occidente durante la segunda ola de feminismos a partir del análisis de la familia nuclear, y de las relaciones que en su seno se daban entre hombres y mujeres, tuvieron -en esos países- enormes consecuencias en el orden social, político, e intelectual. Pero las diferentes perspectivas de análisis -liberal, marxista-socialista, psicoanalítica e incluso post-estructuralista- giraban en torno al análisis crítico sobre las relaciones entre hombres y mujeres en las familias de clase media, nucleares -0 en relación a ellas-, ${ }^{13}$ que no han sido experimentados del mismo modo en los lugares donde la industrialización no ha tenido el mismo calado social o -incluso- por mujeres de clases sociales bajas. Quizá por eso, los feminismos de la segunda ola daban por sentada la existencia de intereses comunes en el grupo de mujeres y el grupo de hombres mientras que los feminismos de la tercera ola no tienen tan clara la focalización en ese grupo social de referencia.

Efectivamente, los feminismos de la segunda ola partían de la teorización de las mujeres como 'esposas' en familias nucleares $-y$ de clase media-, lo que tiene importantes consecuencias (Oyewumi, 2000: 1093-1098). La división de roles en esas familias nucleares -los hombres aportando los ingresos y las mujeres centradas en los trabajos domésticos y de cuidados - está en la base de la división de lo público y lo privado, y de la asignación de los roles sexuales. Pero en África, se afirma, han existido otros modelos familiares. Según Oyewumi, se puede decir que allí las relaciones familiares no se basan en las relaciones matrimoniales, sino en las relaciones consanguíneas, de manera que, "la familia africana no existe como una entidad ligada espacialmente con el hogar desde el momento en que las mujeres pertenecen a sus familias de nacimiento, incluso aunque no residan con ellos" (Oyewumi, 2000: 1096-7). Aunque en las últimas décadas en muchos de esos países se ha producido una fuerte migración del campo a la ciudad, y se han establecido núcleos familiares reducidos en el nuevo espacio urbano, la familia de origen sigue constituyendo una referencia importantísima para mujeres que no encuentran en las leyes de familia un apoyo real que les permita desarrollar con fuerza su propio desarrollo individual al margen de los lazos familiares. Tampoco sus reivindicaciones apuntan hacia un desprendimiento de esos lazos familiares. Lo que denuncian es la falta de recursos económicos para poder contribuir a mejorar la situación de sus familias. ${ }^{14}$

\footnotetext{
${ }^{13}$ Esta misma idea la encontramos en autoras como Donna Haraway, cuando afirma que, "hasta ahora (érase una vez), la encarnación femenina parecía ser dada, orgánica, necesaria, y parecía significar las capacidades de la maternidad y sus extensiones metafóricas” (Haraway, 1995: 309).

${ }^{14}$ En este sentido, no es extraño encontrar a mujeres migrantes que envían dinero a sus países de origen a sus maridos, padres, hermanos e incluso sobrinos. La emigración no significa una desvinculación con la familia de origen o un desarrollo del individualismo. En muchos casos, esto ha sido leído por los investigadores
} 


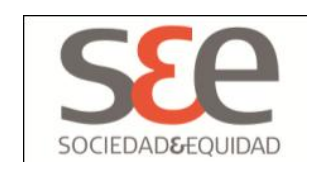

Algo semejante habría que decir de las mujeres occidentales de las clases bajas, del mundo rural, o de aquellas que son madres solteras. Pero quizá, el ejemplo extremo de que las normas sociales respecto a determinadas mujeres no se centra en su rol social como madres lo encontramos en una de las situaciones que Jenny Morris (2008) describe respecto a las mujeres discapacitadas. Este ejemplo pone claramente de manifiesto que, ni siquiera entre las mujeres de clase media de occidente es general el predicamento de que deben ser madres. No para las mujeres discapacitadas, como se deduce de la siguiente situación:

La situación en que un oficial de policía y un asistente social esperaron en el pasillo de un hospital a que una mujer con discapacidad diera a luz, momento en el que le quitaron su bebé de acuerdo con una orden judicial emitida sobre la base de que su discapacidad física le impediría cuidarlo.

(Morris, 2008: 321)

El ejemplo de las mujeres discapacitadas debe ser analizado en este punto, pues a ellas la sociedad les niega incluso la posibilidad de ser madres. Se supone que una mujer discapacitada no es apta para poder cuidar de un bebé. El estereotipo de la discapacitada como asexual, indeseable e incapaz para el amor impide el que estas mujeres puedan ser aceptadas como madres y, también, como cuidadoras (Shakespeare, 1996).

Los feminismos periféricos destacan también el rol crucial de los Estados hegemónicos a la hora de circunscribir las vidas cotidianas de muchas mujeres a luchas por su permanencia y supervivencia, y no se preocupan por considerar únicamente los efectos que produce esa situación en las relaciones familiares de las mujeres. La herencia de esclavitud, la migración forzada, el neocolonialismo, el genocidio y las leyes de extranjería aparecerán como lugares comunes que proyectan criaturas fronterizas, post-coloniales, chicanas o híbridas. Se trata de contar 'historias' que ayuden a subvertir el proceso de otherization (Afshar y Maynard, 2000: 809) que tiene lugar en los países occidentales. ${ }^{15}$ Se aclara que son historias que no caben en los discursos antirracistas, cuyos sujetos son hombres, ni en los discursos tradicionales de género, en los que el sujeto es blanco, ni en el discurso de clase, en el que la raza no tenía lugar (Mirza,

como una reproducción de las desigualdades de género que ya existían antes del proceso migratorio. A nuestro juicio, se trata de mujeres que en muchos casos son conscientes de esas desigualdades pero priorizan el hecho de que sus familias - como totalidad - experimenten una mejora en sus condiciones de vida. Es evidente que el hecho de ser ellas las que aportan los recursos les proporciona también una mejor posición en sus familias, lo que se refleja claramente en su capacidad para tomar decisiones que afectan a cualquier miembro del grupo.

${ }^{15}$ Usamos el término otherization en inglés aunque quizá podríamos usar el castellano alterización. Con ese término las autoras se refieren a un proceso de exclusiones en base a criterios como el sexo, la raza, la religión, la nacionalidad de origen, etc., fruto de la globalización. 


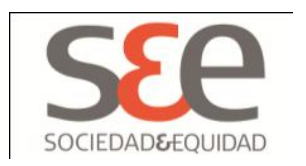

1997:4). Son 'historias' que no aluden a una sola división social, que no vienen justificadas con una Historia.

Por ejemplo, una chicana es alguien que, como señala Anzaldúa, tiene que manejar varias lenguas para sobrevivir entre fronteras. ${ }^{16}$ En un poema que lleva por título 'Una lucha de fronteras / A Struggle of Borders', se empieza a contar la propia 'historia' del siguiente modo, "because I, a mestiza, /continually walk aut of one culture/and into another,/because I am in all cultures at the same time, / alma entre dos mundos, tres, cuatro, / me zumba la cabeza con lo contradictorio. / Estoy norteada por todas las voces que me hablan / simultáneamente" (Anzaldua, 1993). El uso de las dos lenguas, castellano e inglés, reverbera el mestizaje, las pertenencias múltiples y contradictorias que proyectan un tipo de identidad fronteriza.

En las historias de las chicanas destaca la oralidad, y se reivindica una tradición de mujeres que se caracterizan por su falta de acceso a la cultura letrada. La oralidad, y en concreto la expresión mediante poesías, canciones y leyendas, es la manera que estas mujeres eligen para expresar su particular modo de lamentarse (Flores, 2000: 687-705). El mestizaje y la multiplicidad se contemplan como elementos ligados a las posibilidades de resistencia y liberación de las mujeres. Pues,

las personas que cortan-dividen son personas de quienes otras personas se dividenseparan, se disocian, se alejan. Los amantes de la pureza, que controlan mediante la división-separación, no sólo intentan dividirnos-separarnos sino que nos dividen-separan de nosotros mediante (...) la guetización y la exclusión conceptual. También nos intentan dividir-separar de otros que están cortados mediante la lógica de la marginación, de la transparencia. La lógica de la transparencia brilla en la construcción del amante de la pureza, el sujeto moderno, el razonador imparcial. Él es la medida de todas las cosas. Es transparente con relación a su posición en el patriarcado heterorracional, a su cultura, raza, clase y género. Su juicio es el único existente, de manera que los pensamientos cortados son contemplados como algo falto de sentido.

(Lugones, 1999: 260-261)

Las chicanas nos reenvían a las mujeres negras o mujeres de color, que aportan también su historia de 'conciencia opositiva' (Sandoval, 1991: 1-24), es decir, de construcción de afinidades políticas eficaces que no sean réplica de los sujetos revolucionarios imperializantes, que no afrontaron suficientemente en serio las consecuencias de la descolonización. Algunas de estas mujeres de color critican las políticas multiculturalistas que, en nombre de la diversidad, ocultan

\footnotetext{
${ }^{16} \mathrm{El}$ término 'chicana' se refiere a las mujeres de origen mexicano que residen en EEUU, particularmente en California.
} 


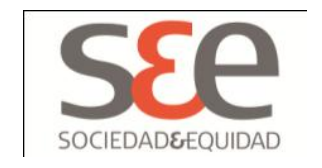

la explotación económica y establecen la centralidad de la cultura anglófona al tiempo que designan a las 'otras culturas' como autónomas y 'subculturas' (Bannerji, 2000 :539).

Podríamos decir que nos hallamos ante identidades con un cierto pasado 'turbio'. La mutilación y el oscurantismo como parte del patrimonio legado aparece explícitamente en algunos casos. Por ejemplo, en los textos de sociólogas marroquíes como Fatima Mernissi, se afirma que querría "tomar la palabra en cuanto mujer marroquí que manipula la escritura y el análisis, dos instrumentos exclusivamente masculinos. $Y$ que no me vengan a contar que en 'nuestro patrimonio ha habido siempre mujeres sabias'. Nuestro patrimonio, tal como lo he vivido de niña, de adolescente y de adulta, es un patrimonio oscurantista y mutilador" (Mernissi, 1991: 30). Desde la India, Ashis Nandi destaca, en la misma línea, que uno de los efectos del neocolonialismo es precisamente la pérdida de la 'virginidad'. Pues a estas alturas no sólo están colonizados los cuerpos sino también las mentes, de manera que las energías se dirigen hacia otros lugares de una vez por todas (Nandy, 1983: XI). Como consecuencia, no se puede esperar ninguna 'autenticidad' de las identificaciones de sexo-género de las mujeres de, en y desde la periferia.

Pero, quizá, el debate de mayor actualidad sobre los efectos del neocolonialismo en las identificaciones de sexo-género de las mujeres del Tercer Mundo nos lo ofrece el análisis de los predicamentos sobre las mujeres de los diferentes grupos islamistas. Por un lado, los movimientos fundamentalistas ${ }^{17}$ en el norte de África, Oriente Próximo y Asia Sudoriental en sus versiones más conservadoras han supuesto un claro retroceso en los derechos de las mujeres. ${ }^{18}$ Para algunos grupos fundamentalistas, la religión y las relaciones tradicionalistas entre hombres y mujeres han llegado a convertirse en un 'recurso étnico' (Anthias y Yuval-Davis, 1993: 4), es decir en un 'material cultural' que dota al grupo de unas credenciales que le hace aparecer ante los otros como merecedores de respeto. Pero esa es sólo una parte de la 'historia'. No todos los movimientos islamistas son necesariamente antifeministas.

El hecho que haya mujeres en los movimientos islamistas obliga a afinar la mirada para tratar de entender a qué se debe su participación. Según Afary, los resultados de las investigaciones de sociólogas como Kandiyoti en Turkía o etnólogas como Ong en Malasia sugieren que, "el impacto de modernización en lo que se refiere a las mujeres ha sido ambivalente. Les ha dado una mayor

\footnotetext{
${ }^{17}$ Como señala Yuval-Davis, por fundamentalismo se solía entender en Gran Bretaña el mantenimiento estricto de las creencias religiosas ortodoxas, tales como la defensa literal de las escrituras sagradas y de las creencias como elementos fundamentales de la cristiandad protestante (Yuval-Davis, 1993). Recientemente, el término se está usando en relación con el contexto de Oriente Próximo, pero también en relación con "corrientes ideológicas similares presentes en todo el mundo, que en las últimas dos décadas han logrado poder político en nombre de la religión, ya se trate del islam, el cristianismo, el judaísmo, el hinduísmo, el budismo o el confucianismo" (Afary, 2000: 47).

${ }^{18}$ Algunas fechas y países que habría que destacar en ese retroceso podrían ir desde la revolución iraní de 1979, pasando por la declaración del Islam como religión del Estado en Bangladesh en 1988, las campañas de terror argelinas lanzadas por el FIS tras ganar las elecciones en 1991 hasta la llegada de los talibanes a Afganistán en 1996.
} 


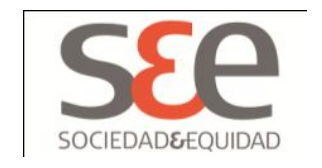

libertad económica y personal como consecuencia del trabajo asalariado, de la disposición de dinero propio para sus gastos y del poder que lo acompaña; pero también ha contribuido a que los hombres abandonaran sus obligaciones tradicionales" (2000:58). En este contexto, defender el islamismo significa defenderse del proceso de individualización que ha afectado a los hombres y que les ha llevado a desentenderse de sus 'familias'. El islamismo es, para ellas, "una misión civilizacional a la vez que les ofrece un protagonismo que su medio tradicional les había negado" (Martín Muñoz, 1997: 76).

La modernización, en esos países, no ha supuesto un aumento de la seguridad diaria y una reducción de las responsabilidades tradicionales de las mujeres. En esas circunstancias, prácticas como pertenecer a un grupo islamista o volver a llevar el hiyab permiten a las jóvenes poder transitar por los espacios públicos sin miedo al acoso sexual, al tiempo que la pertenencia a los grupos islamistas las dota de un reconocimiento social que les permite mantener un mayor espacio de maniobra. Incluso es posible llegar a decir, como hace Nolüfer Göle en su estudio sobre las jóvenes universitarias que portan velo en Turquía, que

tras el velo aparece un nuevo perfil de la mujer musulmana: educada, urbanizada, reivindicativa y que, por el hecho de estar velada no es ni pasiva ni sumisa ni acantonada en el espacio interior. Por tanto, rompe con la imagen de la mujer musulmana tradicional. Por otra parte, contrariamente a la imagen propaganda, no se puede describir al actor islámico simplemente como un marginal, impulsado a la revuelta por el paro y la frustración. Las jóvenes militantes islamistas son también estudiantes universitarias, futuros cuadros, incluso intelectuales.

(Göle, 1991: 11).

Lo anterior no quiere decir, como señalan Afary y Martín Muñoz, que las mujeres musulmanas no desarrollen un discurso feminista. Las propuestas de relectura del Corán son una buena prueba de ello. ${ }^{19}$

\footnotetext{
${ }^{19}$ Afary pone varios ejemplos para demostrar que, "aunque muchas leyes coránicas relativas a las mujeres y a la familia demandan una regulación más conservadora de las relaciones de género, cabría decir que otras se apoyan en las tradiciones matrilineales y matrilocales de la Arabia pre-islámica del siglo VII d. C. Al reconstruir el texto y examinar nuevamente los relatos de la jurisprudencia islámica, las estudiosas feministas utilizan, asimismo, una serie de estrategias legítimas y aceptadas con el propósito de defender sus argumentos. Resaltan los versículos coránicos y los relatos que sugieren un tratamiento más igualitario de las mujeres. Aquellos que imponen restricciones sobre las acciones de las mujeres son reinterpretados" (Afary, 2000: 65). Las conclusiones a las que llegaba Martín Muñoz para las mujeres que pertenecen a los movimientos islamistas en el norte de África son semejantes: "De las encuestas y entrevistas realizadas a estas nuevas veladas del islam, se desprende que entre la variedad de argumentaciones que las islamistas aducen a favor del uso del hiyab (profesionales, feministas, nacionalistas y anti-imperialistas) la religiosa stricto sensu no viene casi nunca sola ni ocupa el primer lugar en el discurso de estas mujeres. De hecho, es
} 


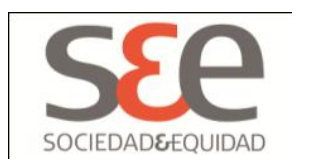

En esas circunstancias, las feministas musulmanas tienen que hacer frente en la actualidad a lo que Mernissi denomina 'terrorismo' antifeminista, que se resume en dos frases; "frase una: 'o que usted está diciendo es una idea importada' (hace referencia al acceso al patrimonio); frase dos: 'lo que usted está diciendo no es representativo' (hace referencia al acceso a la ciencia)" (Mernissi, 1991: 31). Acusadas de traidoras a la causa nacional y reprendidas por poner en cuestión el monopolio de la 'verdad científica' de los hombres de su grupo, mujeres como Mernissi también necesitan distanciarse de lo que sucede en los medios feministas parisienses y neoyorquinos que, dice, analizan las discriminaciones sexuales fuera del contexto de las relaciones de clase y, especialmente, cuando se trata de luchas por el capital simbólico (Mernissi, 1991: 33).

En todo caso, la propuesta de relectura del Corán no es la única posibilidad que se abre para estos feminismos. Otras feministas musulmanas como Haideh Moghissi, presentan argumentos críticos respecto a la modernidad occidental, al tiempo que reivindican un método postmoderno que, sin caer en el relativismo, permita a las mujeres pensar sus propias experiencias en sociedades musulmanas. La metodología postmoderna permite, en este caso, oponerse al argumento según el cual, "el islam y el punto de vista islámico es la única estructura de referencia legítima culturalmente desde la que se pueden reclamar derechos para las mujeres" (Mohissi, 1999: 136).

Los feminismos periféricos todavía están poco articulados con la teoría social. Una propuesta desafiante nos la ofrece desde la India, Gayatri C. Spivak mediante lo que denomina 'estudios subalternos' (Spivak, 1999: 265-300). En su presentación de estos estudios subalternos, Spivak comienza constatando dos procesos de cambio recientes. El primer proceso se refiere al paso del semi-feudalismo al capitalismo monopolista, que ella no considera que deba ser analizado en términos de transición, sino de confrontación de modelos; y el segundo se refiere al cambio funcional en los sistemas de signos, o dicho con nuestras palabras, a la particular manera de presentarse de los actores sociales con capacidad de agencia. Para Spivak, las mujeres del tercer mundo serían el paradigma de la conciencia subalterna pues,

si el campesinado insurgente fue la víctima y el héroe desconocido en la primera ola de resistencia contra el imperialismo territorial (...), es bien sabido que, debido al choque entre las estructuras patriarcales pre-existentes y el capitalismo transnacional, es la mujer sub-proletaria urbana el sujeto paradigmático de la configuración actual de la División Internacional del Trabajo. (Spivak, 1999: 286)

sobre todo su voluntad de 'estar presentes en la sociedad' la que en la práctica se conjuga con el uso vestimentario del hiyab. [...] Para las mujeres islamistas el islam 'verdadero' es el que se inspira en el período de la 'autenticidad', el de la vida del Profeta, porque, después, consideran que los fundamentos islámicos verdaderos fueron progresivamente desviados a causa de la atracción por el poder entre los hombres haciéndose cada vez mayor la distancia entre la práctica socio-política y el dogma religioso. De ahí que se inspiren en el ejemplo de esas mujeres de los primeros tiempos del islam, activas y emprendedoras" (Martín Muñoz, 1997: 77) 


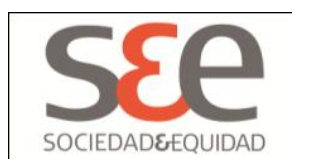

A nuestro juicio, la principal aportación de Spivak consiste en proponer que se vuelva a analizar por qué en países en vías de desarrollo han fracasado los sindicatos socialistas, el capitalismo funcionalista y el comunismo agrario en su intento por desplazar el discurso semi-feudal por un discurso 'moderno'. Las relaciones entre hombres y mujeres, sus cambios y su evolución, representan de manera paradigmática ese fracaso. ${ }^{20} \mathrm{Y}$ por ello, también las condiciones de accesibilidad a un nuevo tipo de conocimiento y a un renovado proceso de cambio social.

Esta astuta propuesta sugiere que profundicemos allí donde aparezcan los efectos de la conciencia negativa o alienada para poder obtener un conocimiento sobre las condiciones de posibilidad para articular algo nuevo. Siguiendo las sugerencias de Spivak, Donna Haraway nos propone abundar en lo que denomina 'la promesa de los monstruos'. Los monstruos serían aquellos agentes sociales que no tienen la capacidad ni el deseo de ser representados en un sujeto colectivo pero que son, sin embargo, capaces de generar con otros su propio relato. Los cyborgs, compuestos de elemento orgánico, técnico, mítico, textual y político no persiguen el progreso, sino la interacción continua, "el placer de la regeneración en zonas fronterizas carismáticas menos devastadoras" (Haraway, 1999: 131).

Lo que se persigue no es representar, sino articular nuevos colectivos a partir de plétoras de actores humanos y no humanos, siendo conscientes de que, "todos estamos en zonas fronterizas quiasmáticas, en áreas liminales en las que se están gestando formas nuevas y tipos nuevos de acción y responsabilidad en el mundo" (Haraway, 1999: 140). Según Haraway, esos nuevos colectivos podrían construirse con la ayuda de artefactos ópticos que estuvieran provistos de filtros rojos, verdes y ultravioletas.

\section{Síntesis final}

Así pues, hemos destacado que los feminismos periféricos se caracterizan por detectar múltiples divisiones sociales y que, por tanto, desechan la idea de que en la sociedad hay dos categorías sociales homogéneas (hombres y mujeres). Hemos puesto de relieve que estos feminismos reivindican unos antecedentes diferentes a los feminismos de la segunda ola, destacando figuras como la de Sojourner Truth, que fue abolicionista al tiempo que feminista. Estos feminismos no resaltan como prioridad la lucha por los derechos legales de las mujeres (la igualdad). Destacan la importancia de la localización y la interseccionalidad. No ponen énfasis en la identidad, sino en las múltiples identificaciones. Podríamos decir que se trata de reivindicaciones de identificaciones relacionales e 'históricas'. Además, estas propuestas no se centran en el modelo occidental de familia nuclear de clase media; resaltan el rol crucial de los estados hegemónicos a la hora de circunscribir las vidas cotidianas de las mujeres a luchas por su

\footnotetext{
${ }^{20}$ Refiriéndose al colectivo de estudios subalternos, Spivak señala que, “en un colectivo que dedica en verdad tanta atención a la subjetividad o a la posición subjetiva del subalterno, resulta sorprendente encontrar tal indiferencia hacia la subjetividad, eso sin mencionar la presencia indispensable de la mujer como instrumento crucial (...). No veo cómo la instrumentalidad crucial de la mujer como objeto simbólico de intercambio puede ignorarse" (Spivak, 1999: 283).
} 


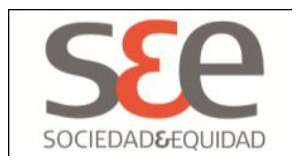

permanencia y supervivencia; destacan la oralidad y no se reivindica una tradición de mujeres que accedieron a la cultura letrada; se trata de identidades con un cierto pasado 'turbio', caracterizado por la mutilación y el oscurantismo; y, por último, suponen posicionamientos concretos en relación con el impacto de la modernización en sus tradiciones culturales y, especialmente, en lo que se refiere a la toma de posición frente a la religiosidad. 


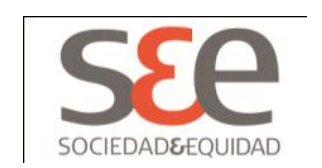

\section{Referencias Bibliográficas:}

Afary, Janet. (2000). La guerra contra el feminismo en nombre del Todopoderoso: reflexiones sobre género y fundamentalismo musulmán. New Left Review, no 6, pp. 45-72.

Afshar, Haleh y Maynard, Mary. (2000). Gender and ethnicity at the millennium: from margin to centre. Ethnic and Racial Studies, Vol. 23, n. 5, pp. 805-819.

Anthias, Floya. (1998). Rethinking social divisions: some notes towards a theoretical framework. Sociological Review, pp. 505-535.

Anthias, Floya y Yuval-Davis, Mira. (1993). Racialized Boundaries. London: Routledge.

Anzaldua, Gloria (1993). La conciencia de la mestiza: Towards a New Conciousness. En Linda S. Kauffman (Ed.), American Feminist Thought at Century's End: A Reader. Oxford: Blackwell Publishers.

Bannerji, Himani. (2000). The construction of a multicultural Canada and 'women of color' en Women's Studies International Forum, Vol. 23, 5, pp. 537-560.

Butler, Judith. (1990). Gender Trouble: Feminism and the Subversion of Identity. New York: Routledge.

Butler, Judith. (2000). Imitación e insubordinación de género, en Revista de Occidente, n. 235, pp. 85-109.

Butler, Judith (2001). Soberanía y actos de habla performativos en Atroposmoderno, http://www.antroposmoderno.com/textos/soberania.shtml

Cole, B.A. (2009). Gender, narratives and intersectionality: can personal experience approaches to research contribute to "undoing gender'? International Review of Education, 55 pp. 561-578.

Flores, Lisa A. (2000). Reclaiming the 'other': toward chicana feminist critical perspective. International Journal of Intercultural Relation, Vol. 24, n. 5, pp. 687-705.

Friedan, Betty. (1974). La mística de la feminidad. Madrid: Ediciones Jucar. (ed. orig. 1963).

Göle, Nilüfer (1991). Musulmanas y modernas. Madrid: Talasa.

Grillo, Trina (1995). Anti-Essentialism and Intersectionality: Tools to Dismantle the Master's House. Berkeley Women's Law Journal, 10, 16-30.

Haraway, Donna (1995). Ciencia, Cyborgs y Mujeres. La reinvención de la naturaleza. Madrid: Cátedra, Col. Feminismos.

Haraway, Donna (1999). Las promesas de los monstruos: Una política regeneradora para otros inapropiados/bles, en Política y Sociedad, no 30, pp. 121-163. 


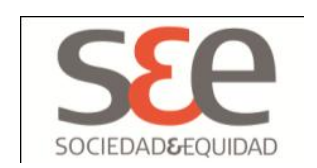

Harding, Sandra (1996): Ciencia y Feminismo. Madrid: Morata. (ed. orig. 1993).

Lewis, Reina y Mills, Sara. (2003). Feminist Postcolonial Theory. A Reader. Edinburgh University Press.

Lorde, Audre. (2003). La hermana, la extranjera. Artículos y conferencias, Madrid, horas y HORAS, la editorial, pp.115-135 (Ed original 1983).

Lugones, María. (1999). Pureza, impureza y separación. En Neus Carbonell y Meri Torras (Comp.) Feminismos literarios. Madrid: Arco Libros, pp. 235-265.

Madoo Lendermann y Niebrige-Brantley. (1993). Teoría Feminista Contemporánea. En George Ritzer, Teoría Sociológica Contemporánea. Madrid: McGraw-Hill, pp. 393-409.

Martín Muñoz, Gema. (1999). Mujeres islamistas, y sin embargo modernas. En Mercedes del Amo (Ed.), El imaginario, la referencia y la diferencia: siete estudios acerca de la mujer árabe. Granada: Departamento de Estudios Semíticos. Universidad de Granada, pp. 75-91.

Mernissi, Fátima. (1992). El miedo a la Modernidad. Islam y Democracia. Madrid: Ediciones de Oriente y del Mediterráneo, Madrid.

Mohanty, C.T. (2008). Bajo los Ojos de Occidente: Feminismo Académico y Discursos Coloniales. En SUÁREZ NAVAZ, L. y HERNÁNDEZ CASTILLO, R. A. (coord.) Descolonizando el Feminismo: Teorías y Prácticas desde los Márgenes. Ed. Cátedra. Madrid. Pp.112-162.

Mirza, Heidi Safia. (ed) (1997). Black British Feminism. London: Routledge.

Moghissi, Haideh. (ed.) (1999). Feminism and Islamic Fundamentalism: The limits of postmodern analysis. London: Zed Books.

Morales, Belén. (2005). Lobby Europeo. Trabajadora, no18, p. 22. Madrid: C.C.O.O. Disponible en http://www.ccoo.es/webCCOO/temp/recursos/1/4431.pdf (Consulta: enero 2010).

Morris, Jenny. (1993). Feminism and Disability. Feminist Review, 43, pp. 57-70.

Morris, Jenny. (2008). Lo personal y lo político. Una perspectiva feminista sobre la investigación de la discapacidad física. En Ler Barton (Comp.) Superar las barreras de la discapacidad. Madrid: Morata, pp. 315-326.

Nandy, Ashis. (1983). The Intimate Enemy. Loss and Recovery of self under colonialism. Oxford: Oxford University Press.

Oyewumi, Oyeronke. (2000). Family Bonds/Conceptual Binds: African Notes on Feminist Epistemologies. Signs, Vol. 25, n. 4, pp. 1093-1098.

Peters, Susan. (1996). La política de la identidad de la discapacidad. En L. Barton (Comp.), Discapacidad y sociedad. Madrid: Morata, pp.230-251. 


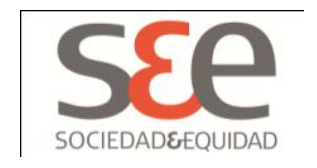

Rich, Adriane. (2003). Notes toward a politics of location. En Reina Lewis y Sara Mills Feminist Postcolonial Theory. A Reader. Edinburgh University Press pp.29-42

Rodríguez Martínez, Pilar. (2002). Hacia una sociología del género y las migraciones: identificaciones de sexo-género de las mujeres migrantes británicas y marroquíes en Almería. Madrid: Universidad Complutense de Madrid.

Rodríguez Martínez, Pilar. (2004). Orden de sexo-género de coerción versus consentimiento: ¿más igualdad real entre hombres y mujeres? Un estudio de contrastes entre poblaciones migrantes. AWAL-Cahiers D'Études Berbères, $n^{\circ} 29$, Éditions de la Maison des Sciences de L'homme. Paris, pp. 65-85.

Rodríguez Martínez, Pilar. (ed) (2006). Feminismos periféricos. Granada: Alhulia.

Rodríguez Martínez, Pilar. (2010). Feminismos y solidaridad. Revista Mexicana de Sociología, 72, n.3, pp. 445-466.

Sa'ar, Amalia. (2005). Postcolonial Feminism, The Politics of Identification and The Liberal Bargain. Gender and Society, Vol.19, No 5, pp. 680-700.

Sandoval, Chela. (1991). Thirt world feminism: The Theory and method of oppositional consciousness in the postmodern world. Gender, 10, pp. 1-24.

Shakespeare, Tom. (1996). Poder y prejuicio: los temas de género, sexualidad y discapacidad. En L. Barton (Comp.), Discapacidad y sociedad. Madrid: Morata, pp.205-230.

Spivak, Gayatri Chakravorty. (1999). Los estudios subalternos: la deconstrucción de la historiografía. En Neus Carbonell y Meri Torras, Feminismos literarios. Madrid: Arco Libros, pp. 265-291.

Talpade Mohanty, Chandra. (2003). Feminism without Borders. Durham and London: Duke University Press.

Truth, Sojourner (1851). Ain't I a Woman?. Disponible en: http://www.fordham.edu/halsall/mod/ sojtruth woman.html (Este texto es parte de Internet Modern History Sourcebook Consulta: mayo 2001). 